\title{
Análisis Tecno-Económico de Gasificación de Polietileno, caso en México
}

\section{Techno-Economic Analysis of Polyethylene Gasification, Mexican case}

\author{
VARGAS-SANTILLÁN, Alfonso $\dagger^{*}$, AGUILAR-GONZALEZ, Alma Leticia, ZUÑIGA-NERIA, \\ Guillermo Capistrano y CASTRO-MONTOYA, Agustin Jaime
}

Instituto Tecnológico Superior de Ciudad Hidalgo

ID $1^{\text {er }}$ Autor: Alfonso, Vargas-Santillán / ORC ID: 0000-0002-9204-3635, CVU CONACYT ID: 445858

ID $1^{\text {er }}$ Coautor: Alma Leticia, Aguilar-González / ORC ID: 0000-0002-0611-1815

ID $2^{\text {do }}$ Coautor: Guillermo Capistrano, Zuñiga-Neria / ORC ID: 0000-0003-0405-4455, CVU CONACYT ID: 446064

ID $3^{\text {er }}$ Coautor: Agustin Jaime, Castro-Montoya / ORC ID: 0000-0002-9206-2553

DOI: $10.35429 /$ JTIP.2019.7.3.1.5

Recibido 03 de Abril, 2019; Aceptado 09 Junio, 2019

\begin{abstract}
Resumen
Como forma de incentivar el uso de nuevas tecnologías para el reciclado de plastico, la investigación obtendrá datos tecno-económicos para el posible reciclo químico de plásticos, la gasificación del polietileno. Se simuló en paquetería ASPEN PLUS para analizar técnica y económicamente la viabilidad del proceso de gasificación, teniendo en cuenta las restricciones y consideraciones de dicho proceso. El desecho principal plástico que se tiene en vertederos son los plásticos Tereftalato de polietileno, Poli estireno y Polietileno de baja y alta densidad. La gasificación es un proceso termoquímico en el que un sustrato carbonoso (en este caso plástico) es transformado en un gas combustible mediante una serie de reacciones que ocurren en presencia de un agente gasificante (generalmente aire) en un ambiente pobre en oxígeno. El trabajo demuestra la viabilidad de este tipo de procesos utilizados en plásticos como lo es polietileno de alta y baja densidad.
\end{abstract}

Gasificación, Polietileno, Aspen Plus

\begin{abstract}
As a way to encourage the use of new technologies for the recycling of plastic, the research will obtain technoeconomic data for the possible chemical recycling of plastics, the gasification of polyethylene. It was simulated in ASPEN PLUS parcel to technically and economically analyze the viability of the gasification process, taking into account the restrictions and considerations of said process. The main plastic waste that is disposed of in landfills are polyethylene terephthalate, polystyrene and low and high density polyethylene. Gasification is a thermochemical process in which a carbonaceous substrate (in this case plastic) is transformed into a combustible gas through a series of reactions that occur in the presence of a gasifying agent (usually air) in an oxygen-poor environment. The work demonstrates the viability of this type of processes used in plastics such as high and low density polyethylene
\end{abstract}

Gasification, Polyethylene, Aspen Plus

Citación: VARGAS-SANTILLÁN, Alfonso, AGUILAR-GONZALEZ, Alma Leticia, ZUÑIGA-NERIA, Guillermo Capistrano y CASTRO-MONTOYA, Agustin Jaime. Análisis Tecno-Económico de Gasificación de Polietileno, caso en México. Revista de Tecnologías en Procesos Industriales. 2019 3-7: 1-5

\footnotetext{
$\dagger$ Investigador contribuyendo como primer autor.
} 


\section{Introducción}

La gasificación de polietileno consiste en la oxidación parcial de las cadenas poliméricas para producir gas de síntesis, mezcla de monóxido de carbono e hidrógeno, que puede ser utilizado como combustible para la generación de electricidad, materia prima para la generación de metano, amoníaco o alcoholes, o como agente reductor para la producción de acero en altos hornos. Esta técnica presenta la ventaja de admitir como alimentación toda la corriente de residuos sin necesidad de separación previa, siendo la técnica más desarrollada de reciclado químico, con plantas comerciales como la de Texaco en E.U.A. y la de Sekundarrohstoff Verwertungs Zentrum (SVZ) en Alemania (Aguado J. S., 2007).

En este proceso se usa aire como agente gasificante, lo que proporciona muchas ventajas. La principal es no usar $\mathrm{O}_{2}$, lo que simplifica el proceso reduciendo el costo; la desventaja es la presencia de $\mathrm{N}_{2}$ (inerte) en el aire que causa reducción en el valor calorífico de los combustibles producidos, debido al efecto de dilución en los gases combustibles al final del proceso. Otro agente gasificante es el vapor de agua, que se puede introducir en relación estequiométrica respecto al $\mathrm{H}_{2}$, evitando la presencia de $\mathrm{N}_{2}$. Varios tipos de procesos de gasificación han sido desarrollados y reportados ampliamente. El principal reto es que durante la gasificación se producen cantidades significativas de carbón, el cual requiere ser procesado y/o quemado. Otros esquemas de gasificación, principalmente a escala piloto, usan gran cantidad de oxígeno, encareciendo el proceso; mientras que otros requieren cantidades considerables de materiales caros como coque y piedra caliza, y depositan mucho lodo de metales difíciles de separar. Un proceso de gasificación ideal para loa plásticos como el polietileno debe producir gas de alto poder calorífico, y no dejar residuos de carbón. Los primeros intentos de gasificación tomando en cuenta los plásticos, fueron publicados desde los 1970s (Buekens, 1978); (Hasegawa, Fukuda, \& Kunii, 1974).

La gasificación en gas combustible de alto poder calorífico obtenido de residuos plásticos se demostró en etapas de investigación, y los resultados se informaron y publicaron en literatura para PVC (Borgianni, Filippis, Pochetti, \& Paolucci, 2002), PP (Xiao, Jin, Zhou, Zhong, \& Zhang, 2007) y PET (Matsunami, Yoshida, Yokota, Nezuka, \& Tamaura, 1999).
Una de las tecnologías más comunes de gasificación, es el proceso Waste Gas Technology UK Limited (WGT) (Figura 9). Con diferentes tipos de desechos (PSW, MSW y lodos) se secan y pretratan mecánicamente, clasificando a las partículas de tamaño óptimo para su posterior alimentación a un reactor cilíndrico de gasificación a (700-900) ${ }^{\circ} \mathrm{C}$, para producir gas de alto poder calorífico (WGT, 2002). Una planta modelo de $500 \mathrm{~kg} / \mathrm{h}$ de capacidad de lodo provenientes de aguas residuales fue instalada por el concesionario OSC Process Engineering Ltd. en el otoño de 1998 para Welsh Water en Nash Water Works; principalmente para operar un sistema de secado a un rango de $110 \mathrm{~kg} / \mathrm{h}$. Además, otra unidad similar se instaló en Francia en 2000.

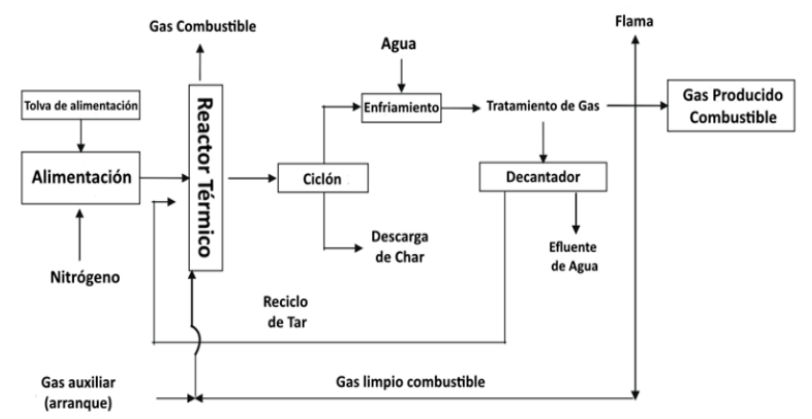

Figura 1 Esquema del proceso WGT

Fuente: (WGT. (2002). Waste Gas Technology Energy from Waste. Available at: http://www.wgtuk.com/ukindex.htmuente)

El proceso de gasificación de Texaco es la tecnología más común de gasificación y la mejor conocida. Los primeros experimentos a escala piloto (10 ton/D) se llevaron a cabo en los Estados Unidos (Weissman, 1997). En el paso de licuefacción, el residuo de plástico es ligeramente desplomerizado térmicamente en compuestos pesados con algunos condensables y fracciones de gas no condensables (Croezen \& Sas, 1997). La gasificación se lleva a cabo con oxígeno y vapor a temperaturas de (1200-1500) ${ }^{\circ} \mathrm{C}$. Después de varios procesos de limpieza (entre otros, eliminación de $\mathrm{HCl}$ y $\mathrm{HF}$ ), se produce gas de síntesis limpio y seco, que consiste principalmente en $\mathrm{CO}$ y $\mathrm{H}_{2}$, con cantidades más pequeñas de $\mathrm{CH}_{4}, \mathrm{CO}_{2}, \mathrm{H}_{2} \mathrm{O}$ y algunos gases inertes. En el caso de residuos plásticos altamente contaminado con otros desechos (incluida la madera contaminada, y tratamiento del lodo de aguas residuales, residuos derivados de industrias petroleras, fracciones de papel, etc.) se conoce al proceso SVZ como la solución óptima.

VARGAS-SANTILLÁN, Alfonso, AGUILAR-GONZALEZ, Alma Leticia, ZUÑIGA-NERIA, Guillermo Capistrano y CASTROMONTOYA, Agustin Jaime. Análisis Tecno-Económico de Gasificación de Polietileno, caso en México. Revista de Tecnologías en Procesos Industriales. 2019 
La entrada se alimenta a un reactor (horno), junto con lignito (en forma de briquetas) y aceite residual. Se utiliza oxígeno y vapor de agua como medios de gasificación, y se suministran en contra flujo con los materiales de entrada (Tukker, de Groot, Simons, \& Wiegersma, 1999). Este proceso genera gas de síntesis, hidrocarburos líquidos y efluentes. Los hidrocarburos líquidos se procesan adicionalmente para formar otros compuestos. El gas se usa principalmente para la producción de metanol y alrededor del $20 \%$ se usa para la producción de electricidad. Una de las principales ventajas de este proceso es su tolerancia a diversos tipos de materia prima a la entrada del proceso.

Las diferentes tecnologías tienen una similitud en la cual la gasificación se basa en que existen diferentes etapas. La primera es un secado, en donde el material plástico es calentado para quitarle cualquier contenido de humedad, este paso también sirve para evaporar el agua y sirva como agente gasificaste. Posteriormente el segundo paso es la pirolisis, en la cual sucede la fragmentación de la molécula polimérica, hasta llegar a hidrocarburos de bajo peso molecular como el metano, etano, etileno, etc.

Por ultimo existe el paso de oxidación y reducción en donde las moléculas obtenidas en el paso anterior reaccionan con un agente gasificaste para la obtención del gas de síntesis.

La tabla 1 muestra las diferentes reacciones propuestas para la obtención de gas de síntesis.

\begin{tabular}{|l|l|l|}
\hline Water-gas-shift & $\mathrm{CO}+\mathrm{H}_{2} \mathrm{O} \leftrightarrow \mathrm{CO}_{2}+\mathrm{H}_{2}$ & -36 (Exo) \\
\hline Water-gas (i) & $\mathrm{C}+\mathrm{H}_{2} \mathrm{O} \leftrightarrow \mathrm{CO}+\mathrm{H}_{2}$ & +136 (Endo) \\
\hline Water-gas (ii) & $\mathrm{C}+2 \mathrm{H}_{2} \mathrm{O} \leftrightarrow \mathrm{CO}_{2}+2 \mathrm{H}_{2}$ & +100 (Endo) \\
\hline Carbon formation & $2 \mathrm{CO} \leftrightarrow \mathrm{C}+\mathrm{CO}_{2}$ & -171 (Exo) \\
\hline Methane formation & $\mathrm{CO}+3 \mathrm{H}_{2} \leftrightarrow \mathrm{CH}_{4}+\mathrm{H}_{2} \mathrm{O}$ & -224 (Exo) \\
\hline Methane formation & $\mathrm{CO}_{2}+4 \mathrm{H}_{2} \leftrightarrow \mathrm{CH}_{4}+2 \mathrm{H}_{2} \mathrm{O}$ & -188 (Exo) \\
\hline
\end{tabular}

Tabla 1 Esquema de reacciones para la producción de gas de sintesis

Fuente: (N.E. Rodriguez et. al. (2015) Simulation of Syngas Production from Lignin Using Guaiacol as a Model Compound Energies 2015, 8, 6705-6714).

Tomando en cuenta los datos tomados en la literatura a cerca de la gasificación de polietileno el presente trabajo muestra el análisis tecno-económico como una propuesta para que se demuestre la viabilidad de dicho proceso.

\section{Metodología a desarrollar}

Se han de identificar las variables que intervienen en el sistema, y que son de interés para el modelo de reciclado químico; éstas se pueden clasificar en:

- Variables exógenas: Aquellas externas al modelo, y que existen con independencia de él; se consideran variables de entrada, y a su vez se pueden dividir en dos grupos:

- Variables controlables o de decisión (factores): Aquellas sobre las que el analista puede decidir su valor, dentro de ciertos límites.

- Variables incontrolables o parámetros: Sus valores no se pueden decidir, sino que vienen fijados. Las variables serán controlables o incontrolables dependiendo de quién las defina.

- Variables endógenas: Aquellas internas, se incluyen las variables de salida del modelo. Son función de las variables exógenas y de la estructura del modelo.

Para el diseño en simulación de polímeros, se tiene una característica diferente a los compuestos convencionales, por ello se necesita introducir diferentes propiedades y la caracterización del componente se tiene que tomar en cuenta. Para ello se ha pensado utilizar las ecuaciones propuestas por Van Krevelen (2009).

Encontrar buenos valores para propiedades físicas faltantes o inadecuadas es la clave para una simulación exitosa; y esto depende de elegir los métodos de estimación correctos. Para llevar cabo adecuadamente una simulación resulta de suma importancia elegir correctamente el método de propiedades que permita inferir el comportamiento de los compuestos durante las reacciones y durante el equilibrio. De acuerdo con cada proceso, se pueden seleccionar diferentes modelos termodinámicos en cada bloque de simulación (Aspen Technology, Inc., 1995).

Las cinco tareas importantes para describir con éxito las propiedades fisicas que se utilizarán en una simulación implican cinco tareas:

1. Seleccionar el método de propiedad física apropiada;

VARGAS-SANTILLÁN, Alfonso, AGUILAR-GONZALEZ, Alma Leticia, ZUÑIGA-NERIA, Guillermo Capistrano y CASTROMONTOYA, Agustin Jaime. Análisis Tecno-Económico de Gasificación de Polietileno, caso en México. Revista de Tecnologías en Procesos Industriales. 2019 
2. Validar las propiedades físicas con datos experimentales;

3. Complementar los componentes en donde no existan datos experimentales (especie o compuesto químico) y parámetros faltantes;

4. Obtener y usar datos de propiedades físicas; y

5. Estimar cualquier parámetro de propiedad faltante.

Se utilizó POLINRTL para las propiedades poliméricas, asi como Peng Robinson cuando se trataba de gases de tipo orgánico. En destiladores y separadores se utilizó UNIFAC. Basado en trabajo de FakhrHoseini en donde utilizó el modelo termodinamico NRTL para predecir las condiciones en equilibrio para las pirólisis de polietileno, polipropileno $\mathrm{y}$ tereftalato de polietileno, en el cual concluyó que el modelo de coeficiente de actividad NRTL podría predecir los productos de la reacción de pirólisis con mucha precisión. (Fakhrhoseini \& Dastanian, 2013).

Como parte de las variables exógenas son las utilizadas en un proceso de gasificación la gasificación implica varios pasos y reacciones químicas complejas, pero se puede resumir en los siguientes pasos: secado, pirólisis, desintegración y reacciones de reformado en la fase gaseosa como se muestra en la figura 2. Una notable ventaja de la gasificación en comparación con los demás procesos es la flexibilidad para valorizar conjuntamente plásticos de diferentes composiciones o mezclas; por tanto las aplicaciones del gas producido dependen del agente gasificante utilizado y no tanto de la composición de la alimentación. La base de calculo para la entrada de del proceso es de $1000 \mathrm{~kg} / \mathrm{hr}$ de polietileno.
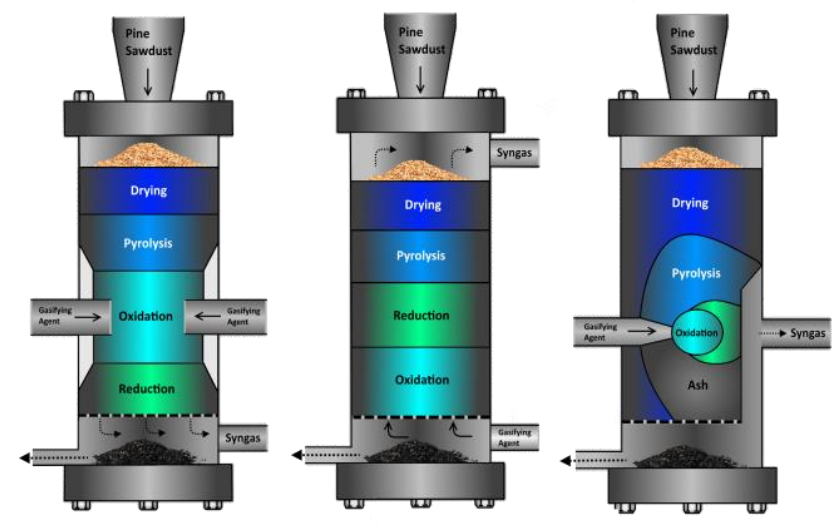

Figura 2 Esquema para el proceso de gasificación

\section{Resultados}

La evaluación técnica de la simulación las variables exógenas tomadas de las fuentes fueron las que se muestra en la tabla 2. Teniendo en cuenta cada etapa para la simulación (Kannan 2011).

\begin{tabular}{|c|c|c|}
\hline Equipo & Factor & Valor \\
\hline \multirow{2}{*}{$\begin{array}{l}\text { Secador/ } \\
\text { Dryer }\end{array}$} & Temperatura & $150^{\circ} \mathrm{C}$ \\
\hline & Presión & $1 \mathrm{~atm}$ \\
\hline \multirow{2}{*}{$\begin{array}{l}\text { Pirólisis/ } \\
\text { RGibbs }\end{array}$} & Temperatura & $600-800^{\circ} \mathrm{C}$ \\
\hline & Presión & $1-7 \mathrm{~atm}$ \\
\hline \multirow{3}{*}{$\begin{array}{l}\text { Reducción y Oxidación } \\
\text { / RGibbs }\end{array}$} & Temperatura & $600-1300^{\circ} \mathrm{C}$ \\
\hline & Presión & $2 \mathrm{~atm}$ \\
\hline & $\begin{array}{l}\text { A. Gasificante/ } \\
\text { Flujo de gas }\end{array}$ & $0-12$ \\
\hline
\end{tabular}

Tabla 2 Especificaciones del proceso de gasificación

Con estos valores se tomó para hacer una optimización para la producción de gas de síntesis donde principalmente es $\mathrm{H} 2$ y $\mathrm{CO}$. Se realizó un análisis de sensibilidad con los diferentes factores para que en base a los resultados en equilibrio en el reactor RGibbs pudiera predecir los mejores valores de operación, los resultados de la obtención de $\mathrm{H} 2$, $\mathrm{CO}, \mathrm{CO} 2$ y $\mathrm{CH} 4$ se muestra en la figura 3.

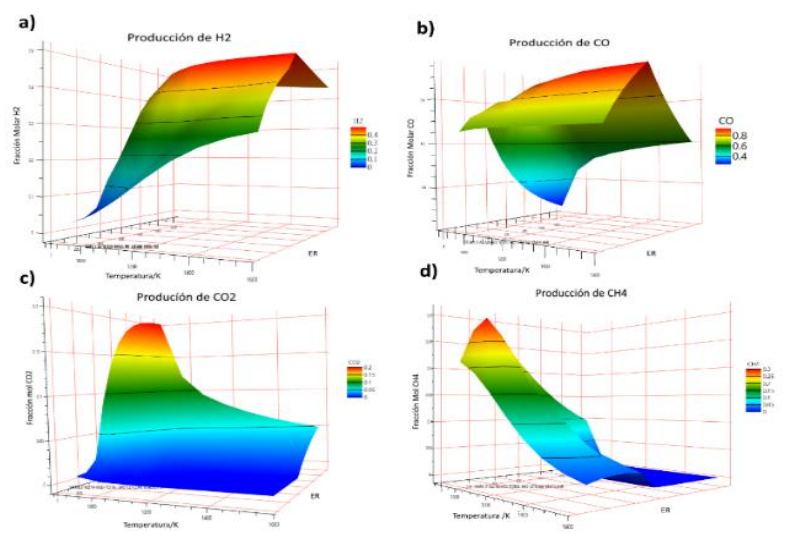

Figura 3 Rendimiento con respecto a la temperatura y la relación de agente gasificaste.

El efecto que tienen las condiciones operativas sobre el rendimiento de la gasificación con aire o vapor. Los parámetros más influyentes son, sin duda, relación de equivalencia (ER) y la temperatura, ya que determina el rendimiento del gas y su composición de H2 (Xiao, Jin, Zhou, Zhong, \& Zhang, 2007). Un aumento en ER conduce a mayores rendimientos de gas rico en hidrogeno (Martínez, Torrico, Pallarés, \& Gil, 2013). 
Como parte indispensable de un proceso, es necesario tener una medida económica de las posibilidades que pueda existir en un escenario hipotético, en este caso en el centro de México. Se tomó una base de cálculo de $1000 \mathrm{~kg} / \mathrm{hr}$ tomando la materia prima del costo de venta de polietileno de baja densidad utilizado de reciclo. Como no existe un precio fijo para el gas de síntesis, ya que es un producto intermediario para diferentes procesos, se toma el precio del monóxido de carbono del mercado actual.

Teniendo los datos anteriores se realiza la simulación y con los datos obtenidos se lleva al programa de Aspen Process Economic Analyzer obteniendo los datos de costos de producción que se muestra en la tabla 3 . Se obtuvo una tasa interna de retorno de $48.3 \%$.

\begin{tabular}{|l|l|l|}
\hline Costo total del proyecto & USD & $\$ 5,642,650.00$ \\
\hline Coste total de funcionamiento & USD & $\$ 5,281,410.00$ \\
\hline Costo Total de Materias Primas & USD & $\$ 3,345,290.00$ \\
\hline Costo Total Servicios & USD & $\$ 1,565,480.00$ \\
\hline Total de Ventas de Productos & USD & $\$ 7,123,270.00$ \\
\hline Precio mínimo de Venta & USD & $\$ 0.89$ \\
\hline
\end{tabular}

Tabla 3 valores de precio mínimo de gas de síntesis

\section{Conclusiones}

Los datos de venta del gas de síntesis no se puede obtener en el mercado, por la limitada forma en que se tiene el transportar el hidrogeno. Los resultados del simulador nos indican que los residuos de polietileno pueden ser utilizados para la producción de gas de síntesis para su posterior utilización. Aun cuando por el momento no puede dar punto de referencia con las tecnologías para la producción de gas de síntesis, es de gran atractivo por el hecho de poder utilizar material de desecho que lo convierte en un potencial recurso de energía.

\section{Referencias}

Aguado, J. S. (2007). European Trends in the FeedstockRecycling of Plastic Wastes.

Aspen Technology, Inc. (1995). ASPEN PLUS User Guide Vol. 1, Release 9. Cambridge, MA : Aspen Technology, Inc.

Borgianni, C., Filippis, P., Pochetti, F., \& Paolucci, M. (2002). Gasification process of wastes containing PVC. Fuel, 1827-1833.
Buekens, A. (1978). Resource recovery and waste treatment in Japan. Recovery and Conservation, 275-306.

Croezen, H., \& Sas, H. (1997). Evaluation of the Texaco gasification process for treatment of mixed household waste. . Netherlands: Final Report of Phase 1 and 2,.

Fakhrhoseini, S., \& Dastanian, M. (2013). Predicting pyrolysis products of PE, PP, and PET using NRTL activity coefficient model. . Hindawi Publishing Corporation, 1-5.

Hasegawa, M., Fukuda, X., \& Kunii, D. (1974). Gasification of solid waste in a fluidized with circulating sand. . Conservation \& Recycling, 143-153.

Kannan Pravin, A.A. (2011). Optimization of Waste Plastics Gasification Process Using Aspen-Plus. INTECH, Chapter 11.

Martínez, L., Torrico, J., Pallarés, J., \& Gil, A. (2013). Thermal valorization of post-consumer film waste in a bubbling bed gasifier. . Waste Manag, 1640-7.

Matsunami, J., Yoshida, S., Yokota, O., Nezuka, M. T., \& Tamaura, Y. (1999). Gasification of waste tyre and plastic (PET) by solar thermochemical process for solar energy utilization. Solar Energy , 21-23.

Tukker, A., de Groot, H., Simons, L., \& Wiegersma, S. (1999). Chemical recycling of plastic waste: PVC and other resins. European Commission, DG III, Final Report. Netherlands: STB-99-55 Final.

Weissman, R. (1997). Recycling of mixed plastic waste by the Texaco gasification process. Cambridge, UK: Chemical Aspects of Plastics Recycling.

WGT. (2002). Waste Gas Technology Energy from Waste. Available at: http://www.wgtuk.com/ukindex.html.

Xiao, R., Jin, B., Zhou, H., Zhong, Z., \& Zhang, M. (2007). Air gasification of polypropylene plastic waste in fluidized bed gasifier. Energy Conversion and Management, 778-786. 